\title{
Synchronized dynamics of Josephson vortices in artificial stacks of SNS Josephson junctions under both dc and ac bias currents
}

\author{
G. R. Berdiyorov, ${ }^{1,2}$ S. E. Savel'ev, ${ }^{1}$ M. V. Milošević, ${ }^{2}$ F. V. Kusmartsev, ${ }^{1}$ and F. M. Peeters ${ }^{2, *}$ \\ ${ }^{1}$ Department of Physics, Loughborough University, Leicestershire, LE11 3TU, United Kingdom \\ ${ }^{2}$ Departement Fysica, Universiteit Antwerpen, Groenenborgerlaan 171, B-2020 Antwerpen, Belgium
}

(Received 15 January 2013; revised manuscript received 2 April 2013; published 28 May 2013)

\begin{abstract}
Nonlinear dynamics of Josephson vortices (fluxons) in artificial stacks of superconducting-normalsuperconducting Josephson junctions under simultaneously applied time-periodic ac and constant biasing dc currents is studied using the time dependent Ginzburg-Landau formalism with a Lawrence-Doniach extension. At zero external magnetic field and dc biasing current the resistive state of the system is characterized by periodic nucleation and annihilation of fluxon-antifluxon pairs, relative positions of which are determined by the state of neighboring junctions. Due to the mutual repulsive interaction, fluxons in different junctions move out of phase. Their collective motion can be synchronized by adding a small ac component to the biasing dc current. Coherent motion of fluxons is observed for a broad frequency range of the applied drive. In the coherent state the maximal output voltage, which is proportional to the number of junctions in the stack, is observed near the characteristic frequency of the system determined by the crossing of the fluxons across the sample. However, in this frequency range the dynamically synchronized state has an alternative-a less ordered state with smaller amplitude of the output voltage. Collective behavior of the junctions is strongly affected by the sloped sidewalls of the stack. Synchronization is observed only for weakly trapezoidal cross sections, whereas irregular motion of fluxons is observed for larger slopes of the sample edge.
\end{abstract}

DOI: $10.1103 /$ PhysRevB.87.184510

PACS number(s): 74.20.De, 74.78.Fk, 74.81.Fa

\section{INTRODUCTION}

Considerable effort has been devoted in the past to the study of the dynamic properties of Josephson vortices (fluxons) in arrays of Josephson junctions (JJs). ${ }^{1-7}$ The interest in the Josephson vortex system is partly due to the possibility of using $\mathrm{J}$ arrays as tunable high-frequency radiation sources, as was for example proposed by Koyama and Tachiki. ${ }^{8}$ Followup experimental ${ }^{9-12}$ and theoretical ${ }^{5,13-15}$ studies have shown that the moving fluxons are responsible for the high-frequency electromagnetic radiation which can open new avenues in the field of superconducting $\mathrm{THz}$ devices (see Refs. 16 and 17 for review). Radiation power generated by the moving vortex lattice was estimated in Ref. 18. In layered JJ systems, the inductive interlayer coupling promotes the formation of the triangular vortex lattice. However, to generate noticeable radiation, oscillations induced by the moving lattice have to be in phase in the different layers, which is realized only when the moving vortices form a rectangular lattice. In this case, the maximal radiation power is proportional to the square of the number of layers in the stack. ${ }^{19}$ Theoretical studies showed that the system mostly contains a triangular Josephson vortex lattice, or similar noncoherent vortex configurations (see, e.g., Ref. 20). In-phase motion of vortices was observed only for specific parameters of the system (i.e., small length and small number of junctions, or higher bias currents and particular magnetic fields).

Therefore, the major challenge in the field is to synchronize Josephson oscillations in all junctions in the stack in order to realize significant radiation. ${ }^{19}$ In-phase oscillations can be achieved by a shunted circuit or by the radiation fields. ${ }^{21,22}$ Effect of microwave irradiation on the properties of JJ arrays have also been studied extensively in the past. For example, the behavior of long JJs in the presence of low-frequency microwave have been studied in relation to their possible application in tuneable resonators, ${ }^{23}$ whereas microwave driven long junctions have been successfully used for phase locking of Josephson flux-flow oscillators in integrated superconducting submillimeter wave receivers. ${ }^{24}$ Particular attention has been given to search for regimes in Josephson flux flow synchronized by the external microwaves. ${ }^{25}$ Under ac drive fluxons show complex dynamic behavior ranging from the synchronized (phase-locked) dynamical regime ${ }^{26-29}$ to the chaotic one. ${ }^{30,31}$ Of special interest are the dynamic properties of JJs in the presence of external ac and dc forcing. Intriguing transport phenomena have been reported in recent years, including absolute negative conductance (i.e., a positive dc current can generate a negative voltage across the junction) ${ }^{32}$ and/or negative nonlinear conductance. ${ }^{33}$ A different level of complexity is added to the context when the neighboring junctions have different parameters. A typical example is the stack of JJs of different length realized, e.g., in mesa structures from high-temperature superconductors, which present natural stacks of atomistic-scale intrinsic JJs. ${ }^{12,34}$ Since the junctions oscillate with different frequencies in different stacks, it is difficult to put the whole system into a coherent state. $^{35}$

In this work we study the effect of a nonrectangular cross section of an artificial stack of superconducting-normalsuperconducting (SNS) JJs on the dynamics of fluxons under time-periodic ac and constant dc biasing currents. We show that very small deviations of the sidewalls of the system from its vertical position strongly affect the collective behavior of the junctions, resulting in a transition from coherent motion of fluxons to less ordered dynamics of fluxons. No phase synchronization is observed for larger asymmetry in the distribution of junction cross sections, where the dynamic state is characterized by a chaotic motion of fluxons. 


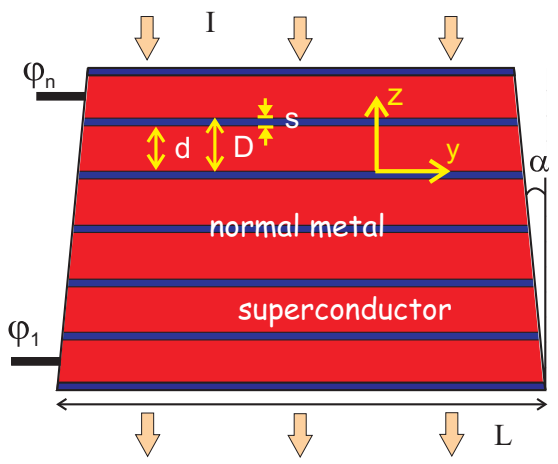

FIG. 1. (Color online) Model system: a stack of superconducting layers (of thickness $d$ ) separated by normal metal Josephson junctions (of thickness $s$ ) in the presence of uniform external current $I$ applied through normal metal contacts. The angle $\alpha$ determines the length of each junction. Horizontal black stripes show the position of the potential contacts that measure the output voltage between the layers 1 and $n$.

\section{THEORETICAL APPROACH}

We consider artificial stack of superconducting layers of thickness $d$ separated by uniform normal metal junctions (thickness $s$ ) in the presence of a uniform external current $I$ applied through the normal contacts at the top and bottom of the sample (see Fig. 1). The system has nonvertical sidewalls, where the lateral size of the sample is determined by the angle $\alpha$. To understand the dynamic properties of the system we used the time dependent Ginzburg-Landau (TDGL) formalism and solved numerically the following TDGL equation inside each of the superconducting layers:

$$
u\left(\frac{\partial}{\partial t}+i \varphi\right) \psi=(\nabla-i \mathbf{A})^{2} \psi+\left(1-|\psi|^{2}\right) \psi+\chi,
$$

where the last term is a small white noise, the size of which is much smaller than the barrier height between the different metastable states. The Josephson tunneling between the superconducting layers is taken into account using the following coupling (see the Appendix):

$$
\begin{aligned}
& \left.\left(-i \nabla_{z}-A_{z}\right) \psi_{n}\right|_{\text {top boundary }} \\
& =\frac{i}{\mu s}\left[\psi_{n+1}(x, y,(n+1) D)\right. \\
& \left.\quad \times \exp \left(-i \bar{A}_{n+1} s\right)-\psi_{n}(x, y, n D+d)\right], \\
& \left.\left(-i \nabla_{z}-A_{z}\right) \psi_{n}\right|_{\text {bottom boundary }} \\
& =\frac{i}{\mu s}\left[\psi_{n-1}(x, y,(n-1) D+d)\right. \\
& \left.\quad \times \exp \left(i \bar{A}_{n-1} s\right)-\psi_{n}(x, y, n D)\right],
\end{aligned}
$$

on top [Eq. (2a)] and bottom [Eq. (2b)] of each superconducting layer, where $d$ and $s$ are the thickness of the superconducting and metallic layers, respectively, and $D=d+s$. Here $\bar{A}$ is defined as $\bar{A}_{n+1} \equiv(1 / s) \int_{n D+d}^{(n+1) D} A_{z} d z$ and $\mu$ is the ratio of the mass of the Cooper pairs in the metallic and superconducting regions. The superconducting-vacuum boundary condition $\left.\vec{a}(-i \nabla-\mathbf{A}) \psi\right|_{a}=0$ is applied in other boundaries with $\vec{a}$ being the unit vector normal to the surface. Additionally, we use de Gennes boundary condition $\left.{ }^{36}(-i \nabla-\mathbf{A}) \psi\right|_{a}=\left.i \psi\right|_{a}$ at the superconducting-normal boundaries, to account for the proximity effect. Equation (1) is coupled with the equation for the electrostatic potential $\varphi$ :

$$
\sigma \Delta \varphi=\operatorname{div}\left(\mathbf{j}_{\mathbf{s}}\right),
$$

which follows from the continuity equation:

$$
\partial \rho / \partial t=\nabla \cdot\left[\mathbf{j}_{\mathrm{s}}-\sigma(\nabla \varphi+\partial \mathbf{A} / \partial t)\right],
$$

with assumptions that the charge relaxes fast (i.e., no charge accumulation) and the variation of the magnetic field over time is smaller than the other physical processes in the system. The superconducting current is given by

$$
\mathbf{j}_{\mathrm{s}}=\frac{1}{2 i}\left(\psi^{*} \nabla \psi-\psi \nabla \psi^{*}\right)-\mathbf{A}|\psi|^{2},
$$

with a Josephson component $j_{s \perp}$ (see the Appendix):

$$
\begin{aligned}
j_{s \perp}= & \frac{1}{2 i \mu s}\left(\psi_{n+1}(x, y,(n+1) D)\right. \\
& \times \exp (-i \bar{A} s) \psi_{n}^{*}(x, y, n D+d) \\
& \left.-\psi_{n+1}^{*}(x, y,(n+1) D) \exp (i \bar{A} s) \psi_{n}(x, y, n D+d)\right) .
\end{aligned}
$$

Assuming that the superconductor has a good contact with the normal-metal current contacts at the top and bottom of the sample, we set $\psi=0$ at the contacts and inject the external current through the boundary condition for the electrostatic potential $\left.\nabla \varphi\right|_{a}= \pm j$, with $j$ being the applied current density. Since the superconducting current is zero at the contacts, the current is injected to the system in the form of normal current, which then converts to the superconducting one over the distance of less than few coherence length (see, e.g., Ref. 37). In these equations, we express the length in units of the coherence length $\xi$ and the vector potential is scaled to $\Phi_{0} /(2 \pi \xi)$ (where $\Phi_{0}$ is the magnetic flux quantum). Time is in units of the Ginzburg-Landau relaxation time $t_{0}=4 \pi \lambda^{2} / c^{2} \rho_{n}$ ( $\rho_{n}$ is the normal-state resistivity), the electrostatic potential is in units of $V_{0}=c \Phi_{0} \rho_{n} / 8 \pi^{2} \lambda \xi$, and the current density is measured in $j_{0}=c \Phi_{0} / 8 \pi^{2} \lambda^{2} \xi$. The parameter $u$, which is a measure of the different relaxation times (e.g., the relaxation time of the absolute value of the order parameter) in the superconductor, is taken as $u=5.79 .{ }^{38}$ Using $\xi(0)=10 \mathrm{~nm}$, $\lambda(0)=200 \mathrm{~nm}$, and $\rho_{n}=18.7 \mu \Omega \mathrm{cm}$, which are typical for $\mathrm{Nb}$ thin films, ${ }^{39}$ we obtain $t_{0} \approx 2.69$ ps and $V_{0}=0.12 \mathrm{mV}$ close to the critical temperature. We assume that the junctions are unform in the $x$ direction, which reduces the problem to two dimensions. We also neglected the demagnetization effects which are valid when the lateral dimensions of the sample are much smaller than the Pearl length $\Lambda=2 \lambda^{2} / d$. We put the conductivity $\sigma=1$ in the simulations. These coupled nonlinear differential equations are solved self-consistently in the $y-z$ plane using explicit Euler (for $\psi$ ) and multigrid (for $\varphi)$ iterative procedures. Equation (1) is discretized using the link variable approach (see, e.g., Ref. 40). To avoid extremely time consuming simulations (due to the explicit simulation method) we consider a limited number of junctions in the stack $(N<10)$ and the results are presented here for zero external magnetic field. 


\section{DC BIASING-EFFECT OF NONVERTICAL CROSS SECTIONS}

We begin our analysis by demonstrating the properties of our system in the presence of applied dc current by constructing the time-averaged voltage versus applied current $(I-V)$ and the voltage versus time $(V(t))$ characteristics of the sample. As a representative example, we consider stacks of six superconducting layers (of thickness $d=10.5 \xi$ and base length $L=256 \xi$ ) separated by $N=5$ metallic junctions of thickness $s=0.5 \xi$, the $I-V$ characteristics of which are shown in Fig. 2 for three different values of the slope angle $\alpha$. We first discuss the results for $\alpha=0$ (solid black curve), which is the situation when all the junctions have the same length. For the given parameters of the junctions, zero resistance of the sample is maintained up to a threshold current density $j_{c}=0.09 j_{0}$, above which the system transits into a resistive state with a finite-voltage jump. Notice that this critical current is much smaller than the Ginzburg-Landau depairing critical current $\left(\sim 0.385 j_{0}\right)$, which corresponds to the penetration of Abrikosov vortices in uniform thin film superconductors. ${ }^{41}$ Since we work in the zero external magnetic field regime, the dissipation arises from the periodic nucleation and annihilation of Josephson vortex-antivortex pairs as shown in panels (1a) and (1b) of Fig. 2 where we plotted snapshots of the
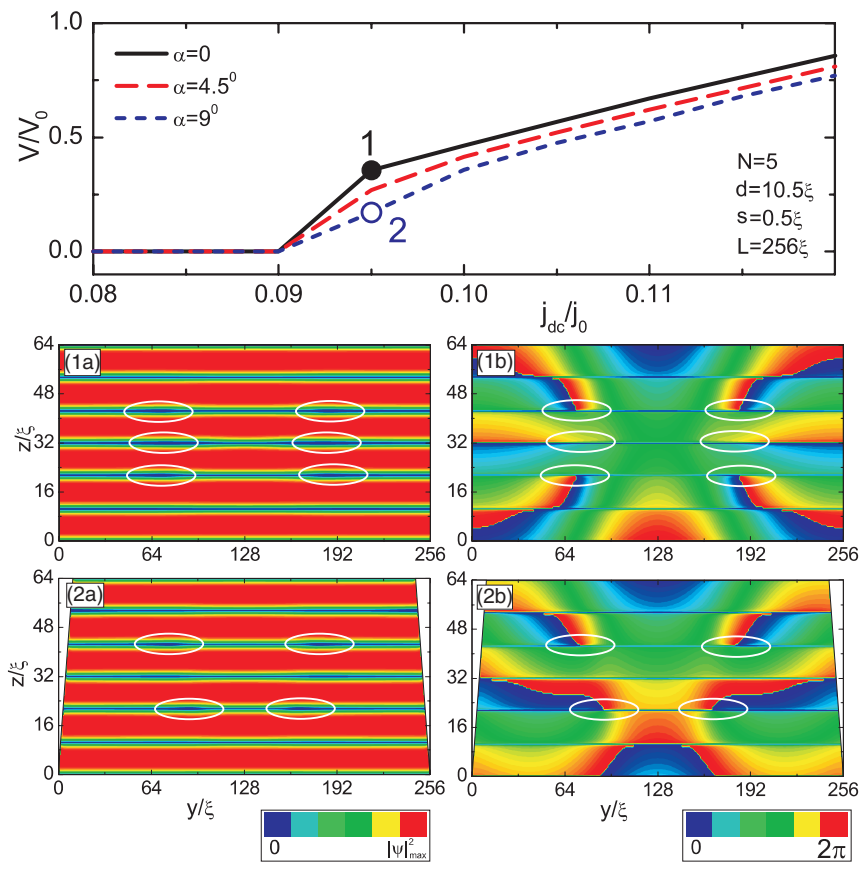

FIG. 2. (Color online) Time averaged voltage ( $V$, measured across the whole sample) vs current density $(j)$ characteristics of the sample with five stacked junctions $(N=5)$ at zero applied magnetic field for tilting angles $\alpha=0$ (solid black curve), $\alpha=4.5^{\circ}$ (dashed red curve), and $\alpha=9^{\circ}$ (dotted blue curve). The base length of the sample is $L=256 \xi$, the thickness of the superconducting layer is $d=10.5 \xi$, and the thickness of the normal layer is $s=0.5 \xi$. Panels (1a), (1b), (2a), and (2b) show snapshots of the Cooper-pair density [(1a),(2a)] and the phase of the order parameter $[(1 b),(2 b)]$ at $j=0.95 j_{0}$ for $\alpha=0[(1 a),(1 b)]$ and $\alpha=9^{\circ}[(2 a),(2 b)]$ indicated by the points 1 and 2 on the $I-V$ curves. White circles indicate the positions of the Josephson vortices and antivortices.
Cooper-pair density (1a) and the phase of the order parameter (1b). ${ }^{42}$ The locations of fluxons are highlighted by white circles. The nucleation rate of the fluxon-antifluxon pairs increases with increasing applied current resulting in a monotonic increase of the output voltage. Dashed-red and dotted-blue curves in Fig. 2 show the $I$ - $V$ curves of the sample for finite slope of the sidewalls. Although the output voltage decreases with increasing the tilting angle $\alpha$, the critical current density of the sample does not depend on the junction cross section. For the considered values of the tilting angle, the entire sample switches into the resistive state when the critical current is reached. This resistive state is still characterized by the periodic nucleation of fluxon-antifluxon pairs in each junction [see panels (2a) and (2b) in Fig. 2].

To show properties of the resistive state of the system, and the role of the mutual interactions between the fluxons in neighboring junctions, we plotted in Fig. 3 the time evolution of the output voltage for the sample of Fig. 2 with $\alpha=0$, together with snapshots of the Cooper-pair density. We have chosen the small dc current regime to retain a single fluxon-antifluxon mode and to avoid possible self-heating at
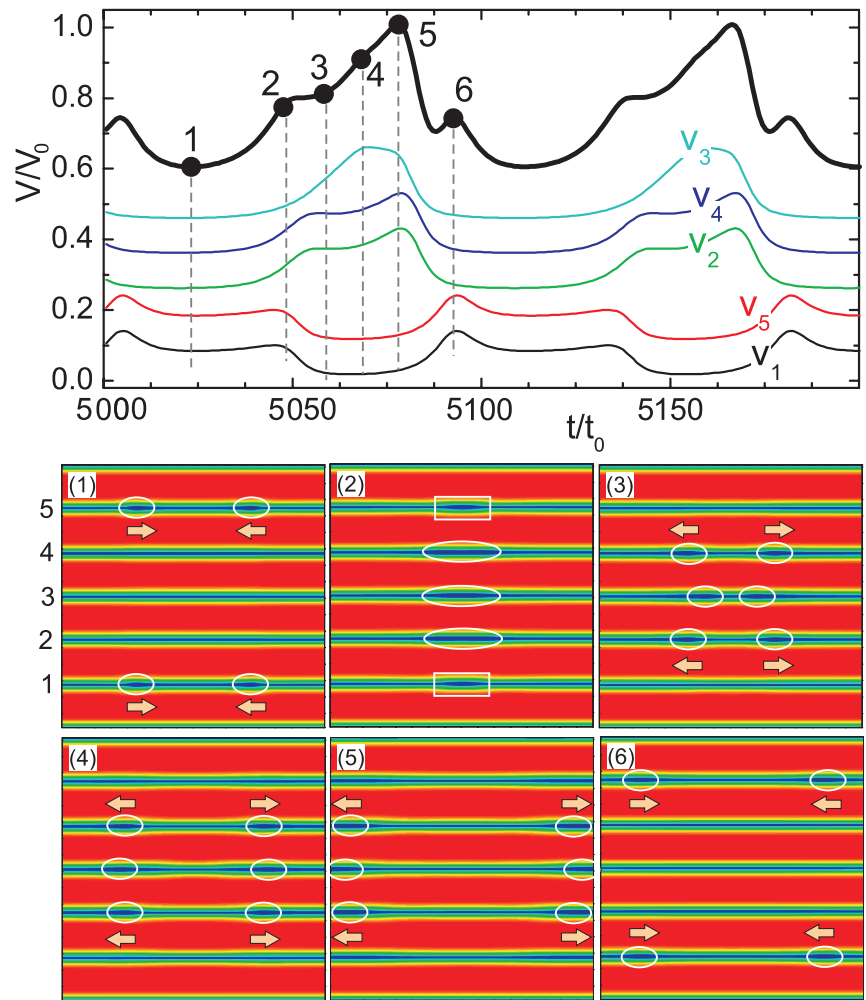

FIG. 3. (Color online) Voltage vs time characteristics of the sample in Fig. 2 for $\alpha=0$ and $j=0.095 j_{0}$. Voltage is calculated across the whole sample (thicker black curve) and across each junction indicated by $V_{1}, \ldots, V_{5}$ [see left axis of panel (1) for indexing of the junctions]. To avoid overlap of the curves the results are shifted by $0.1 V_{0}$ for $V_{5}, 0.25 V_{0}$ for $V_{2}, 0.35 V_{0}$ for $V_{4}, 0.45 V_{0}$ for $V_{3}$, and $0.4 V_{0}$ for the voltage curve across the sample. Panels (1)-(6) show snapshots of $|\psi|^{2}$ at the times indicated by circles in the main panel. Arrows indicate the direction of motion of Josephson vortices (from right to left) and antivortices (from left to right). Larger circles/rectangles in panel (2) show the Josephson vortex-antivortex nucleation point and rectangles indicate their annihilation in the middle of the sample. 
larger velocities of the fluxons. The $V(t)$ characteristics of the sample show periodic oscillations (two periods are shown here) with several peaks in each period (see thicker black curve in the main panel of Fig. 3), which originates from the periodic nucleation and annihilation of fluxon-antifluxon pairs in different layers. For the given set of sample parameters and applied current these pairs oscillate with characteristic period $T^{*}=88.5 t_{0}$ common for all the junctions. Although, in-phase motion of fluxons in neighboring junctions is energetically unfavorable due to the repulsive interaction between the fluxons, the geometry of the sample induces synchronization in the motion of fluxons in selected pairs of junctions. In this particular case, junctions 1 and 5 oscillate in phase as shown in panel 1 of Fig. 3 (the same is observed between junction 2 and 4). As a consequence, we observe an identical voltage signal across these junctions (see curves $V_{1}$ and $V_{5}$; notice the vertical shift of the voltage curve $V_{5}$ ). Two maxima in these curves correspond to the nucleation (at the edge of the junctions; see panel 1) and annihilation (in the middle of the sample; panel 2) of fluxon-antifluxon pairs. The latter process creates a favorable condition for the nucleation of fluxon-antifluxon pairs in the middle of the sample in neighboring junctions (see panel 2). Once nucleated, these pairs move towards the edges of the sample (panels 3 and 4) where they leave the sample (panel 5). Each nucleation and annihilation process results in local maxima in the voltage across the junction, which contributes to the total voltage signal. Such breather-type selfoscillations have been recently proposed to be the mechanism of emission from spatially uniform stacked JJs at zero magnetic field. ${ }^{43}$

Figure 4 shows the $V(t)$ characteristics of the sample for the case of a finite slope of the sidewalls $\left(\alpha=4.5^{\circ}\right)$. The applied current density is just above the critical current. Snapshots of $|\psi|^{2}$ (bottom figures of Fig. 4) illustrate the motion of the fluxons. As in the case of a uniform sample (Fig. 3) the voltage signal arises from the periodic nucleation and annihilation of the Josephson vortex-antivortex pairs (panels 1-3). However, in contrast to the symmetric sample, the nucleation point of the pairs in the same junction can alter in time from the

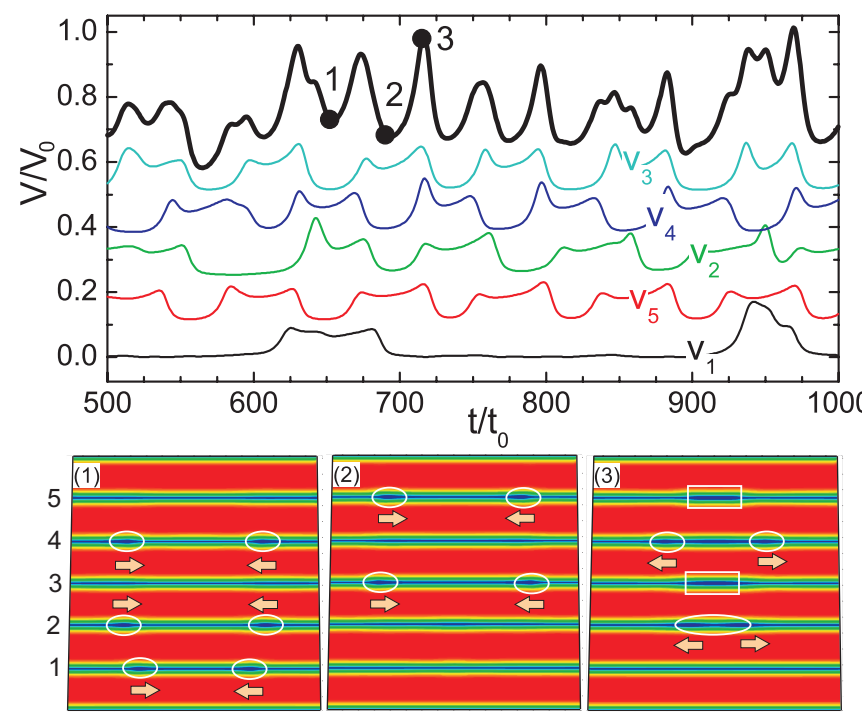

FIG. 4. (Color online) Same as in Fig. 3 but now for $\alpha=4.5^{\circ}$. middle of the sample to the edges depending on the state of the neighboring junction (see the second junction in panels 1 and 3). ${ }^{44}$ Notice that no crossing of fluxon-antifluxon pairs are observed due to the stronger dissipation in our system as compared to sine-Gordon systems. The motion of fluxons in different junctions are strongly irregular, which is clearly seen from the voltage curves $V_{1}-V_{5}$ measured across each junction. For larger values of the angle $\alpha$, some of the junctions switch to the zero voltage state (not shown here) resulting in a more chaotic voltage signal across the sample.

Thus the resistive state of an artificial stack of JJs at zero magnetic field is characterized by the periodic nucleation and annihilation of Josephson vortex-antivortex pairs, the positions of which are determined by the flux state of the neighboring junctions. In-phase oscillations of selected pairs of junctions can be observed in dc currents due to the symmetry of the system. Small deviation of the sidewalls of the sample from the vertical position puts the stack into the incoherent state in which junctions oscillate at different frequencies.

\section{SYNCHRONIZED DYNAMICS OF FLUXONS IN THE PRESENCE OF AC CURRENT}

In this section we investigate the dynamics of fluxons in the stack of JJs when the system is subjected to both a time-periodic (and spatially uniform) ac current and a constant biasing dc current (also spatially uniform). Our main goal is to realize in-phase oscillations of all the junctions in the stack by synchronizing the fluxons by an external ac forcing. As an example, we consider sinusoidal ac plus static dc current biasing, so that the total current density is given by

$$
j=j_{\mathrm{dc}}+\left|j_{\mathrm{ac}}\right| \sin (2 \pi t / T),
$$

where $T$ is the period of the current oscillations. We restrict ourselves to lower dc currents (just above the resistive state transition current $j_{c}$ ) to retain the single-fluxon-antifluxon regime in each junction. We also consider a weak ac signal such that no fluxons will be generated by the ac current. This is to avoid complex dynamical effects observed when large amplitude ac forcing induces a flow of fluxons in the system (see, e.g., Ref. 31).

First we consider the symmetric sample with rectangular cross section with the parameters as in Fig. 2. Figure 5 shows the voltage vs time response of the sample in the presence of ac current with different period $T$ and with dc component $j_{\mathrm{dc}}=0.095 j_{0}$. As a reference, we plotted in Fig. 5(a) the $V(t)$ curve of the sample with only dc current, which was discussed in Fig. 3. Although small in amplitude $\left(\left|j_{\mathrm{ac}}\right|=0.1 j_{\mathrm{dc}}\right)$, the ac component of the current results in considerable modulation of the voltage signal across the sample: strongly modulated voltage peaks are observed in every half-period of the ac signal [Fig. 5(b)] the amplitude of which is much larger than the voltage signal at dc biasing. Due to difficult temporal adjustment between the fluxon motion and the driving force, small voltage peaks are observed at the beginning of the second reverse cycle. ${ }^{45}$ The number of voltage peaks decreases in the half-cycle and their amplitude increases with increasing frequency of the ac current [Figs. 5(c)-5(e)]. Note that all the results in Fig. 5 are plotted with the same $y$-axis scale. The maximal output voltage is observed when half of the period of 

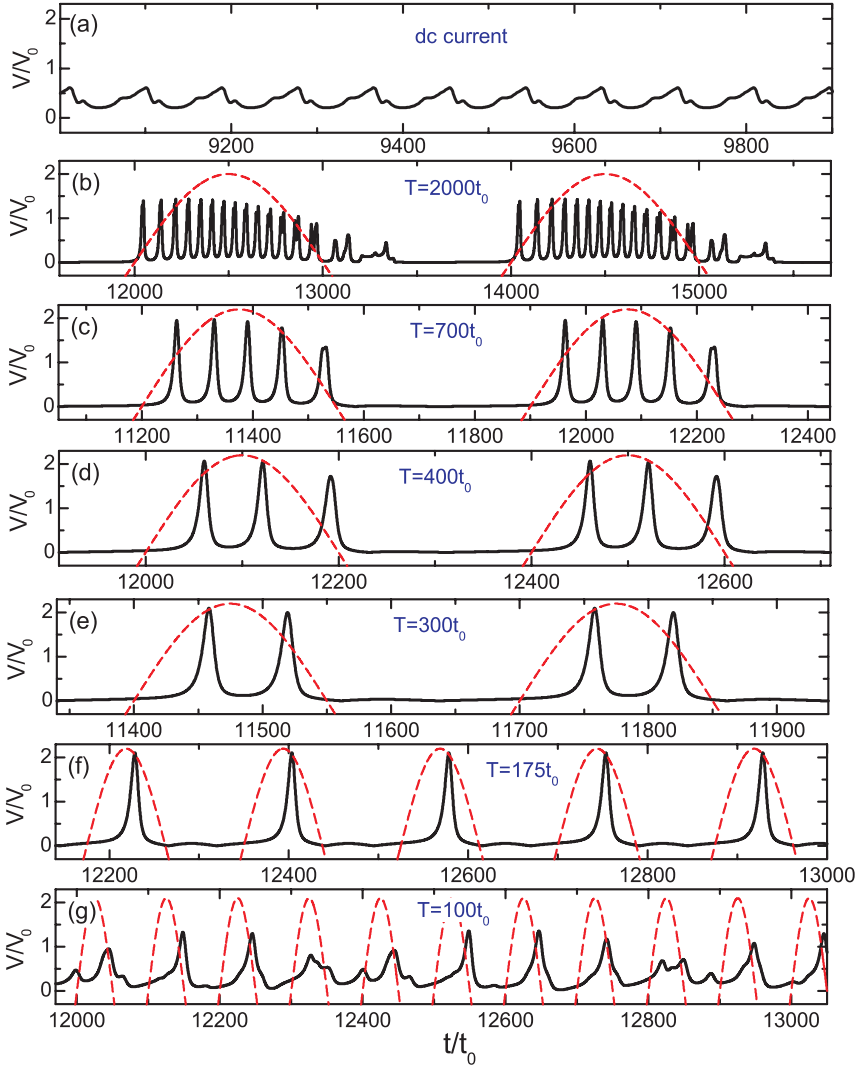

FIG. 5. (Color online) $V(t)$ curves of the sample of Fig. 2 in the presence of dc (a) $\left(j_{\mathrm{dc}}=0.095 j_{0}\right)$ and ac (b) $-(\mathrm{g})\left[j=0.095 j_{0}+\right.$ $\left.0.0095 j_{0} \sin (2 \pi t / T)\right]$ currents. The period of the ac drive is $T=$ $2000 t_{0}$ (b), $T=700 t_{0}$ (c), $T=400 t_{0}$ (d), $T=300 t_{0}$ (e), $T=175 t_{0}$ (f), and $T=100 t_{0}(\mathrm{~g})$. Dashed-red curves illustrate the profile of the ac component of the current.

the ac signal approaches the characteristic period of the system $T^{*}=88.5 t_{0}$ determined by the dynamics of the fluxons for the given value of the dc current. The voltage curve of the sample in this case is shown in Fig. 5(f). The shift in the phase of the ac signal and the voltage response of the system is due to the finite relaxation time of the superconducting order parameter. During the half-period of the current oscillation, fluxons on one side of the sample and antifluxons on the other side are injected simultaneously in all junctions and propagate through the junction under the influence of the dc bias current retaining the same $y$ coordinate (i.e., rectangular lattice). Further increase of the frequency of the external field results in less ordered Josephson flux-flow motion and a reduction of the voltage signal [Fig. 5(g)].

Figure 6 shows the Fourier power spectra of the voltage curves of Fig. 5: $\tilde{V}(v)=\frac{1}{\sqrt{2 \pi}} \int V(t) \mathrm{e}^{i \frac{2 \pi t}{T}} d t$. dc current regime [Fig. 6(a)] is characterized by a pronounced peak in the $|\tilde{V}(v)|^{2}$ curve with the characteristic frequency $v^{*}=1 / T^{*}$ of the periodic oscillations of fluxon-antifluxon pairs. Extra peaks in the Fourier spectrum appear with adding the ac component to the applied current current [Fig. 6(b)]. The amplitude of these peaks increases and the number of the peaks decreases with increasing frequency of the applied drive [Figs. 6(c)-6(e)] indicating a stronger modulation of the Josephson oscillations. The latter is also evident from

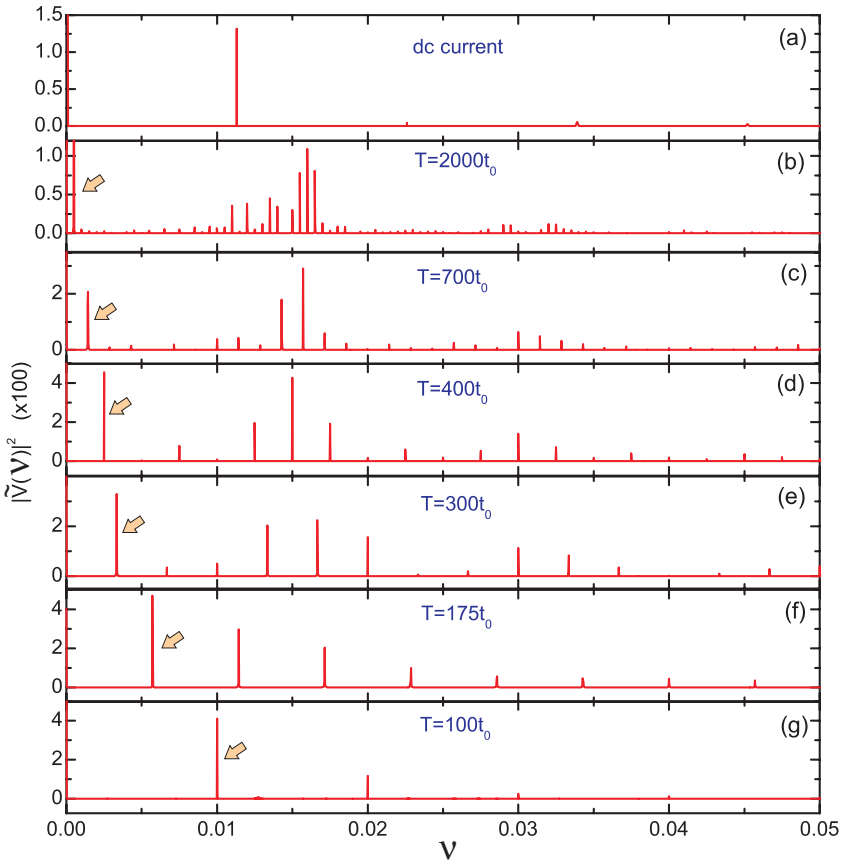

FIG. 6. (Color online) Fourier power spectrum $|\tilde{V}(v)|^{2}$ of the voltage curves in Fig. 5. Voltage oscillations with the frequency of the external ac drive $v^{*}=1 / T^{*}$ are indicated by arrows.

the $V(t)$ curves of the system [see Figs. 5(c)-5(e)]. Largest peak in the spectrum with clearly visible harmonics is seen in Fig. 6(f), where the motion of fluxons is synchronized with the frequency of the ac drive. When the frequency of the ac current is larger than the characteristic frequency of the system, we find only peaks in the spectrum corresponding to the frequency of the external drive (and some of its harmonics) as shown in Fig. 6(g). Note that phase-locked dynamics of the fluxons is observed for all the frequencies of the applied drive with corresponding peaks in the Fourier spectra indicated by arrows in Fig. 6.

Thus the present simulation results show that applying a small time-periodic ac component to the dc current acts as an effective restoring force between the fluxons in the different junctions, thereby countering their mutual repulsion. Synchronization of all the junctions into a macroscopic coherent state is observed when the frequency of the ac drive approaches the characteristic frequency of the system, determined by the dynamics of the fluxons. Similar results were found for a larger number of junctions in the stack (i.e., $N=10)$.

In what follows, we study the effect of a nonrectangular cross section of the stack of junctions on the dynamics of fluxons when the system is subjected to both a timeperiodic ac and a constant biasing dc current. Figure 7 shows the voltage vs time curves of the sample with base length $L=256 \xi$ and $N=5$ junctions in the stack under the constant $\mathrm{dc}\left(j_{\mathrm{dc}}=0.095 j_{0}\right)$ and sinusoidal ac $\left(\left|j_{\mathrm{ac}}\right|=\right.$ $\left.0.1 j_{\mathrm{dc}}\right)$ currents for different periods of the ac drive. In the case of dc biasing, voltage oscillations are strongly irregular [Fig. 7(a)], and the oscillation spectrum is very broad, resembling a chaotic state [Fig. 8(a)]. This is because each junction oscillates at slightly different frequencies affected by the 

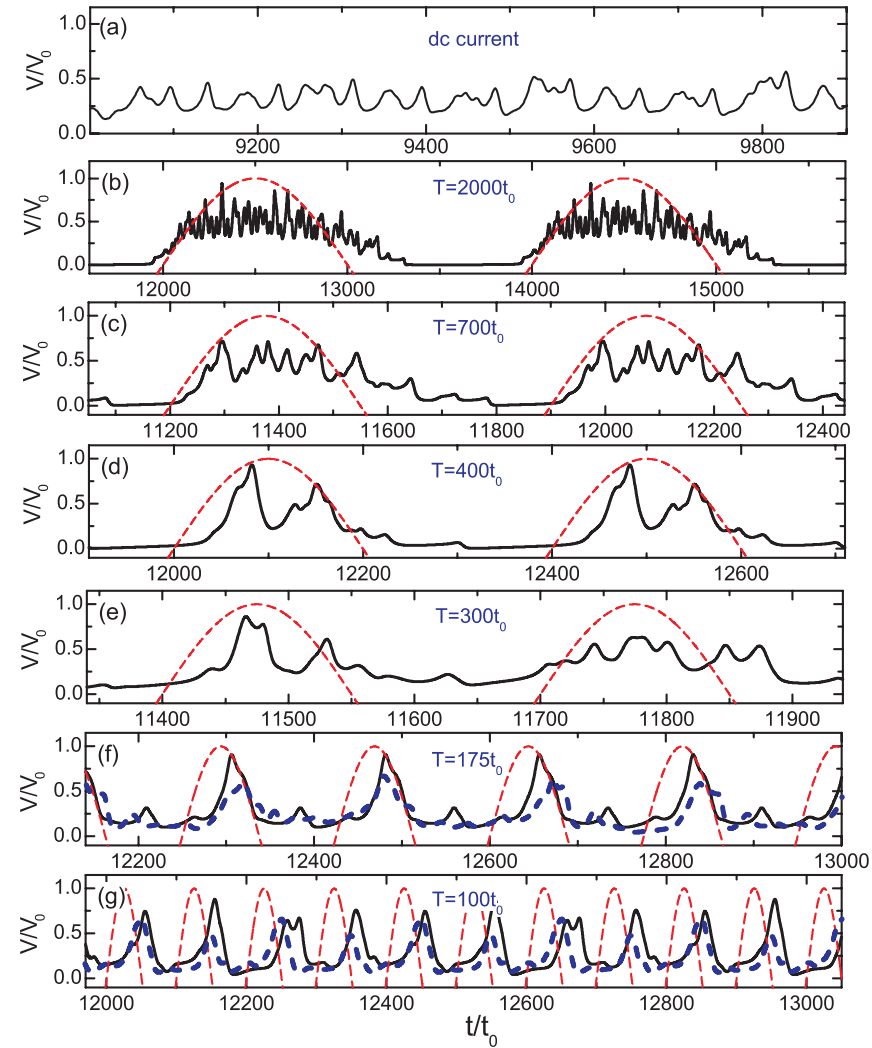

FIG. 7. (Color online) Same as in Fig. 5 but for the tilting angle $\alpha=4.5^{\circ}$. Dotted-blue curves in (f) and (g) show the $V(t)$ curve of the sample for $\alpha=9^{\circ}$.

length of the junction. The voltage oscillates with an average characteristic frequency $v^{*} \approx 0.023 / t_{0}$, which is modulated by low frequency oscillations [see the peaks in Fig. 8(a)]. In-phase motion of the fluxons is unfavorable without the ac drive. At lower frequencies of the ac current, a bunch of fluxon-antifluxon pairs is injected at every half-period, the motion of which becomes irregular in the junctions. This is clearly seen in the $V(t)$ curve of the sample [Fig. 7(b)]. In the Fourier spectrum many additional, well separated peaks are observed [Fig. 8(b)]. Their motion becomes more regular with increasing frequency of the applied current, as evidenced by sharp peaks in the spectrum [see Figs. 8(c)-8(e)]. The most ordered state is shown in Fig. 7(f) for $T=175 t_{0}$. However, the amplitude of the output voltage is twice smaller than the one for the uniform sample [compare Figs. 5(f) and 7(f)], which indicates that not all the junctions in the system oscillate in phase. Moreover, due to difficult temporal adjustment between the fluxon motion and the driving force during the cycle, we observed an extra peak in the other half-period of the current oscillations [Fig. 7(f)]. Figure 7(g) shows the voltage curve of the sample when the frequency of the oscillations is larger than the characteristic frequency of the system. Phase-locked dynamics of the fluxons with the applied drive is seen from this figure. This is also clearly seen in the corresponding Fourier spectrum presented in Fig. 8(g).

The dotted-blue curve in Fig. 7(f) shows the voltage vs time characteristics of the sample for a larger slope of the sidewalls $\left(\alpha=9^{\circ}\right)$. It is seen from this figure that the peaks in the voltage curve are not very much pronounced and its

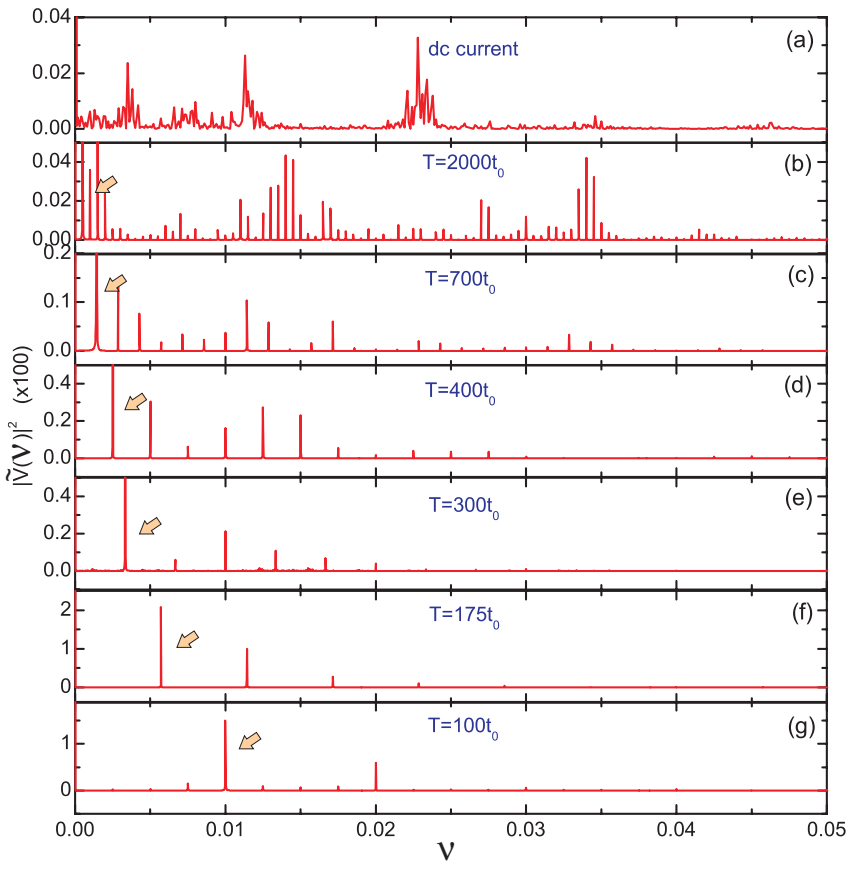

FIG. 8. (Color online) Fourier power spectrum $|\tilde{V}(v)|^{2}$ of the voltage curves in Fig. 7. Voltage oscillations with the frequency of the external ac drive are indicated by arrows.

peak value is considerably reduced. This is a consequence of the out-of-phase motion of fluxons in different junctions. However, phase-locked oscillations become more pronounced at larger frequencies of the ac current [see dotted blue curves in Fig. $7(\mathrm{~g})]$.

Thus the dynamics of Josephson vortices under dc current becomes irregular when the slope of the sample's sidewall are changed. Their motion can be synchronized by applying a small amplitude ac current only for smaller values of the tilting angle.

To summarize our findings in this section, we plotted in Fig. 9 the amplitude of the voltage oscillations as a function of the period of ac current for three different values of the angle $\alpha$. The results are normalized to the amplitude of the voltage signal of the dc current. For a uniform sample ( $\alpha=0$, circles) considerable increase of the voltage signal is observed already for a larger period of the ac current. However, the spectrum of the voltage oscillations consists of several well separated peaks [see Fig. 6(b)]. The amplitude of the voltage signal increases further with increasing frequency of the ac current and reaches its maximum when the frequency of the drive is in the range of the characteristic frequency of the system. All the junctions oscillate in phase and the voltage signal becomes proportional to the number of junctions in the stack. However, this synchronized state coexists with a less ordered state resulting in the formation of a "gap" between synchronized and unsynchronized states. The system can transit between these two states due to, e.g., thermal fluctuations as shown in Fig. 9(b), where we plotted the voltage vs time characteristics of the sample for $T=175 t_{0}$. The inset highlights the dynamical transition from the partially synchronized state [characterized by many oscillating frequencies in the spectrum; see Fig. 9(c)] to a completely synchronized state 


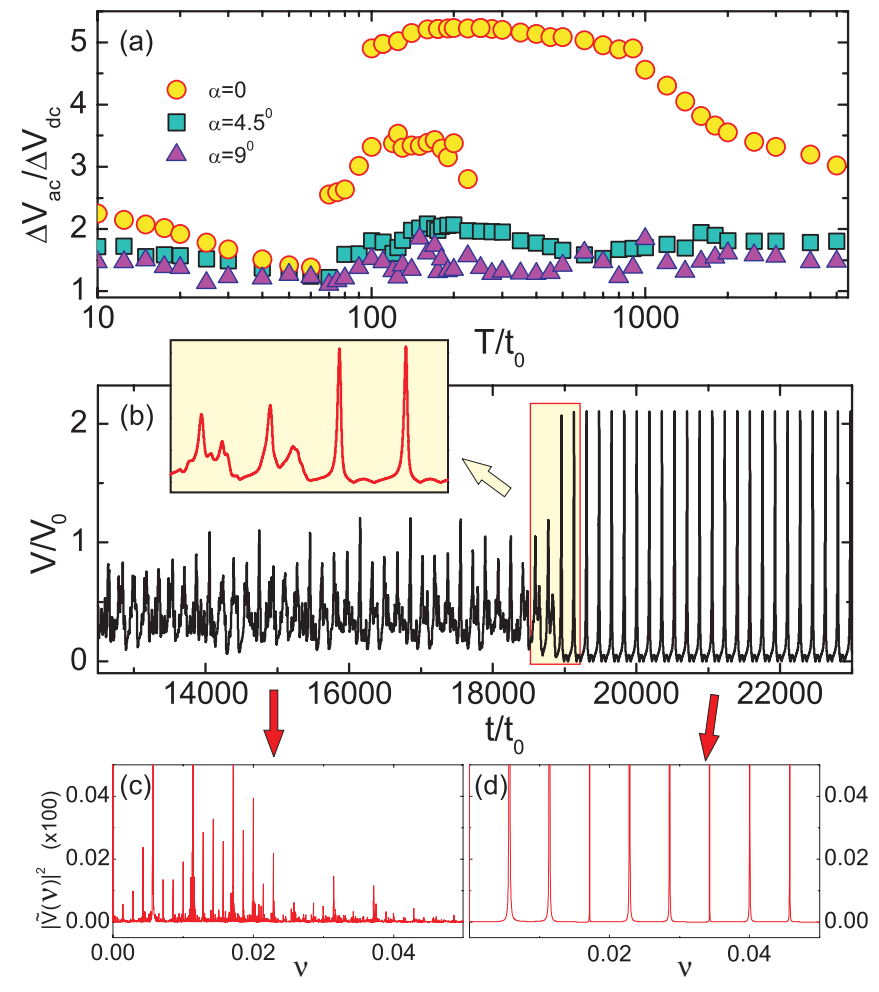

FIG. 9. (Color online) (a) Amplitude of the voltage oscillations as a function of the period $T$ of the sinusoidal applied current $j=j_{\mathrm{dc}}+$ $\left|j_{\mathrm{ac}}\right| \sin (2 \pi t / T)$ with $j_{\mathrm{dc}}=0.095 j_{0}$ and $\left|j_{\mathrm{ac}}\right|=0.1 j_{\mathrm{dc}}$ for the slope angle $\alpha=0$ (circles), $\alpha=4.5^{\circ}$ (squares), and $\alpha=9^{\circ}$ (triangles). The base length of the sample is $L=256 \xi$, the thickness of the superconducting layers is $d=10.5 \xi$, the thickness of the normal metal layer is $s=0.5 \xi$, and the number of junctions in the stack is $N=5$. (b) $V(t)$ curves of the sample for $\alpha=0$ and $T=175 t_{0}$. The inset shows the transition point between nonregular to synchronized Josephson oscillations. (c), (d) The Fourier power spectrum $|\tilde{V}(v)|^{2}$ of the voltage curves of the sample with $\alpha=0$ for the period of the ac current $T=175 t_{0}$ when the system is in the chaotic state (c) and in the synchronized state (d), indicated by red vertical arrows.

[with well separated peaks in the spectrum; see Fig. 9(d)]. At larger frequencies moving fluxons phase lock to the applied drive leading to considerable increase of the voltage signal. The amplitude of the voltage signal drops considerably with changing slope of the sample sidewalls, as shown by the square and triangular symbols in Fig. 9(a). However, partial synchronization can be observed for smaller shallowness of the sidewall profile at the frequencies close to the averaged characteristic frequency of the system. Phase-locked oscillations of the junctions to the applied drive are observed at higher frequencies even for larger tilting angles.

\section{CONCLUSION}

We study the dynamics of Josephson vortices in artificial stacks of SNS Josephson junctions in the presence of timeperiodic ac and constant biasing dc currents. At zero applied magnetic field and with a biasing dc current the fluxons nucleate in pairs either at the edges of the junctions or in the middle of the sample depending on the state of the neighboring junctions. Namely, the annihilation process of the fluxon-antifluxon pairs in a given junction stimulates the nucleation of another pair in the neighboring junction. Adding a small ac component to the dc current results in synchronized motion of fluxons in all the junctions forming a rectangular lattice in spite of their mutual repulsive interactions. The maximal output signal, which is proportional to the number of junctions in the stack, is observed when the frequency of the ac drive equals the characteristic frequency of the system, determined by the dynamics of the fluxons. However, in this frequency range the dynamically stabilized state coexists with the less ordered state but with a smaller amplitude of the output voltage. Collective behavior of the junctions is strongly affected by the sloped walls of the system: phase synchronization is observed only for a smaller deviation of the distribution of the junction cross sections, whereas irregular motion of fluxons is observed in the case of a larger slope of the sample edge.

\section{ACKNOWLEDGMENTS}

This work was supported by the Flemish Science Foundation (FWO-Vl) and by EU Marie Curie (Project No. 253057).

\section{APPENDIX: GENERALIZED LAWRENCE-DONIACH MODEL}

Let us consider a stack of superconducting layers of finite thickness $d$ separated by Josephson-coupled insulating layers of thickness $s$ (see Fig. 10). The free energy of the system can be expressed as ${ }^{46}$

$$
\begin{aligned}
F_{s}= & \sum_{n} \int d x d y\left[\int _ { n D } ^ { n D + d } d z \left(\frac{\hbar^{2}}{2 m^{*}}\left|\left(\nabla-\frac{i e^{*}}{\hbar c} \mathbf{A}\right) \psi_{n}(x, y, z)\right|^{2}+\alpha\left|\psi_{n}(x, y, z)\right|^{2}+\frac{\beta}{2}\left|\psi_{n}(x, y, z)\right|^{4}\right.\right. \\
& \left.\left.+\frac{\hbar}{2 m_{z}^{*} s^{2}}\left|\psi_{n+1}(x, y,(n+1) D) \exp \left(-\frac{i e^{*}}{\hbar c} \bar{A}_{n+1} s\right)-\psi_{n}(x, y, n D+d)\right|^{2}\right)\right]+\frac{1}{8 \pi} \int d x d y d z B^{2}(x, y, z)
\end{aligned}
$$

where $\bar{A}_{n+1} \equiv(1 / s) \int_{n D+d}^{(n+1) D} A_{z}(x, y, z) d z$ and $D=d+s$. Contrary to the original proposal of Lawrence and Doniach, ${ }^{47}$ where the order parameter is defined only on the $n$th superconducting plane, i.e., $\psi_{n}=\psi_{n}(x, y)$, here $\psi_{n}(x, y, z)$ is a continuous function of the coordinates inside the $n$th superconducting layer. 


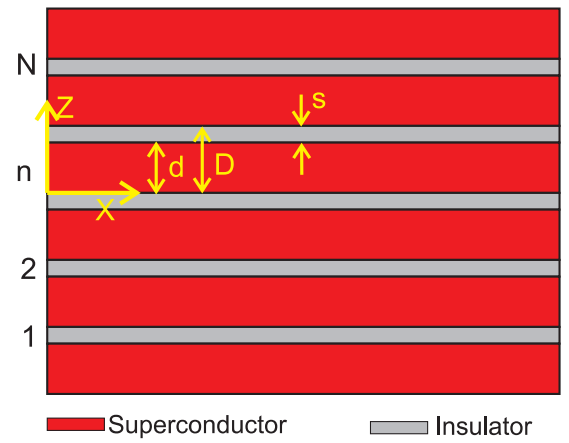

FIG. 10. (Color online) Schematic representation of an SIS Josephson tunnel junction.

Variation of this free energy expression with respect to $\psi_{n}^{*}$ results in the following Ginzburg-Landau equation:

$$
\begin{aligned}
& \alpha \psi_{n}(x, y, z)+\beta\left|\psi_{n}(x, y, z)\right|^{2} \psi_{n}(x, y, z) \\
& +\frac{1}{2 m^{*}}\left(-i \hbar \nabla-\frac{e^{*}}{c} \mathbf{A}\right)^{2} \psi_{n}(x, y, z)=0
\end{aligned}
$$

inside the $n$th superconducting layer and the following boundary conditions for the $n$th layer:

$$
\begin{aligned}
& \left.\left(-i \frac{\partial}{\partial z}-\frac{e^{*}}{c \hbar} A_{z}\right) \psi_{n}\right|_{\text {top boundary }} \\
& =\frac{i}{\mu s}\left(\psi_{n+1}(x, y,(n+1) D)\right. \\
& \left.\quad \times \exp \left(-\frac{i e^{*}}{\hbar c} \bar{A}_{n+1} s\right)-\psi_{n}(x, y, n D+d)\right),
\end{aligned}
$$

$$
\begin{aligned}
& \left.\left(-i \frac{\partial}{\partial z}-\frac{e^{*}}{c \hbar} A_{z}\right) \psi_{n}\right|_{\text {bottom boundary }} \\
& =\frac{i}{\mu s}\left(\psi_{n-1}(x, y,(n-1) D+d)\right. \\
& \left.\quad \times \exp \left(+\frac{i e^{*}}{\hbar c} \bar{A}_{n-1} s\right)-\psi_{n}(x, y, n D)\right),
\end{aligned}
$$

which takes into account for the Josephson coupling between the superconducting layers, and

$$
\left.\vec{a}\left(-i \nabla-\frac{e^{*}}{c \hbar} \mathbf{A}\right) \psi_{n}\right|_{\text {other boundaries }}=0,
$$

where $\vec{a}$ is the unit vector normal to the surface of the superconductor and $\mu=m_{z}^{*} / m^{*}$.

Variation of Eq. (A1) with respect to $\mathbf{A}$ results in the following expression for the supercurrent density:

$$
\begin{aligned}
\mathbf{j}_{s}= & -\frac{i \hbar e^{*}}{2 m^{*}}\left[\psi_{n}^{*}(x, y, z) \nabla \psi_{n}(x, y, z)-\psi_{n}(x, y, z) \nabla \psi_{n}^{*}(x, y, z)\right] \\
& -\frac{e^{*}}{m^{*} c}\left|\psi_{n}(x, y, z)\right|^{2} \mathbf{A},
\end{aligned}
$$

with the Josephson component,

$$
\begin{aligned}
\mathbf{j}_{s \perp}= & -\frac{i \hbar e^{*}}{2 m_{z}^{*} s}\left(\psi_{n+1}(x, y,(n+1) D)\right. \\
& \times \exp \left(-\frac{i e^{*}}{\hbar c} \bar{A} s\right) \psi_{n}^{*}(x, y, n D+d) \\
& -\psi_{n+1}^{*}(x, y,(n+1) D) \\
& \left.\times \exp \left(+\frac{i e^{*}}{\hbar c} \bar{A} s\right) \psi_{n}(x, y, n D+d)\right),
\end{aligned}
$$

which tunnels between layers $n$ and $n+1$. *francois.peeters@ua.ac.be

${ }^{1}$ R. Kleiner, Phys. Rev. B 50, 6919 (1994).

${ }^{2}$ A. V. Ustinov and S. Sakai, Appl. Phys. Lett. 73, 686 (1998).

${ }^{3}$ M. Machida, T. Koyama, A. Tanaka, and M. Tachiki, Physica C 330, 85 (2000).

${ }^{4}$ T. Clauss, T. Uchida, M. Mößle, D. Koelle, and R. Kleiner, Appl. Phys. Lett. 85, 3166 (2004).

${ }^{5}$ M. Tachiki, M. Iizuka, K. Minami, S. Tejima, and H. Nakamura, Phys. Rev. B 71, 134515 (2005).

${ }^{6}$ H. B. Wang, S. Urayama, S. M. Kim, S. Arisawa, T. Hatano, and B. Y. Zhu, Appl. Phys. Lett. 89, 252506 (2006).

${ }^{7}$ S. O. Katterwe and V. M. Krasnov, Phys. Rev. B 80, 020502 (2009).

${ }^{8}$ T. Koyama and M. Tachiki, Solid State Commun. 96, 367 (1995).

${ }^{9}$ G. Hechtfischer, R. Kleiner, A. V. Ustinov, and P. Müller, Phys. Rev. Lett. 79, 1365 (1997).

${ }^{10}$ Yu. I. Latyshev, M. B. Gaifullin, T. Yamashita, M. Machida, and Y. Matsuda, Phys. Rev. Lett. 87, 247007 (2001).

${ }^{11}$ J. Zitzmann, A. V. Ustinov, M. Levitchev, and S. Sakai, Phys. Rev. B 66, 064527 (2002).
${ }^{12}$ L. Ozyuzer, A. E. Koshelev, C. Kurter, N. Gopalsami, Q. Li, M. Tachiki, K. Kadowaki, T. Yamamoto, H. Minami, H. Yamaguchi, T. Tachiki, K. E. Gray, W.-K. Kwok, and U. Welp, Science 318, 1291 (2007).

${ }^{13}$ E. Goldobin, A. Wallraff, N. Thyssen, and A. V. Ustinov, Phys. Rev. B 57, 130 (1998).

${ }^{14}$ S. Savel'ev, V. Yampol'skii, A. Rakhmanov, and F. Nori, Phys. Rev. B 72, 144515 (2005).

${ }^{15}$ F. Marchesoni, S. Savel'ev, M. Tachiki, and F. Nori, Phys. Rev. B 81, 174531 (2010).

${ }^{16}$ S. Savel'ev, V. A. Yampol'skii, A. L. Rakhmanov, and F. Nori, Rep. Prog. Phys. 73, 026501 (2010).

${ }^{17}$ X. Hu and S.-Z. Lin, Supercond. Sci. Technol. 23, 053001 (2010).

${ }^{18}$ L. N. Bulaevskii and A. E. Koshelev, Phys. Rev. Lett. 97, 267001 (2006).

${ }^{19}$ A. K. Jain, K. K. Likharev, J. E. Lukens, and J. E. Sauvageau, Phys. Rep. 109, 309 (1984).

${ }^{20}$ S. Madsen and N. F. Pedersen, Phys. Rev. B 72, 134523 (2005); C.-Yu Liu, G. R. Berdiyorov, and M. V. Milošević, ibid. 83, 104524 (2011). 
${ }^{21}$ S.-Z. Lin, X. Hu, and L. Bulaevskii, Phys. Rev. B 84, 104501 (2011).

${ }^{22}$ I. Martin, G. B. Halász, L. N. Bulaevskii, and A. E. Koshelev, J. Appl. Phys. 108, 033908 (2010).

${ }^{23}$ E. Goldobin, A. M. Klushin, M. Siegel, and N. Klein, J. Appl. Phys. 92, 3239 (2002).

${ }^{24}$ V. P. Koshelets and S. V. Shitov, Supercond. Sci. Technol. 13, R53 (2000).

${ }^{25}$ G. Costabile, R. Monaco, S. Pagano, and G. Rotoli, Phys. Rev. B 42, 2651 (1990).

${ }^{26}$ N. F. Pedersen and A. Davidson, Phys. Rev. B 41, 178 (1990).

${ }^{27}$ M. Salerno, M. R. Samuelsen, G. Filatrella, S. Pagano, and R. D. Parmentier, Phys. Rev. B 41, 6641 (1990).

${ }^{28}$ N. Grønbech-Jensen, Phys. Rev. B 47, 5504 (1993); Phys. Lett. A 169, 31 (1992); N. Grønbech-Jensen and M. Cirillo, Phys. Rev. B 50, 12851 (1994); M. Cirillo, P. Cocciolo, V. Merlo, N. GrønbechJensen, and R. D. Parmentier, J. Appl. Phys. 75, 2125 (1994).

${ }^{29}$ S. Madsen and N. Grønbech-Jensen, Phys. Rev. B 71, 132506 (2005).

${ }^{30}$ M. Cirillo, J. Appl. Phys. 60, 338 (1986); M. Cirillo, A. R. Bishop, N. Grønbech-Jensen, and P. S. Lomdahl, Phys. Rev. E 49, R3606 (1994).

${ }^{31}$ F. L. Barkov, M. V. Fistul, and A. V. Ustinov, Phys. Rev. B 70, 134515 (2004).

${ }^{32}$ L. Machura, M. Kostur, P. Talkner, J. Luczka, and P. Hänggi, Phys. Rev. Lett. 98, 040601 (2007).

${ }^{33}$ M. Kostur, L. Machura, P. Talkner, P. Hänggi, and J. Luczka, Phys. Rev. B 77, 104509 (2008).
${ }^{34}$ M.-H. Bae, H.-J. Lee, and J.-H. Choi, Phys. Rev. Lett. 98, 027002 (2007).

${ }^{35}$ T. M. Benseman, A. E. Koshelev, K. E. Gray, W.-K. Kwok, U. Welp, K. Kadowaki, M. Tachiki, and T. Yamamoto, Phys. Rev. B 84, 064523 (2011).

${ }^{36}$ P. G. de Gennes, Superconductivity of Metals and Alloys (AddisonWesley, New York, 1994).

${ }^{37}$ T. Y. Hsiang and J. Clarke, Phys. Rev. B 21, 945 (1980).

${ }^{38}$ L. Kramer and R. J. Watts-Tobin, Phys. Rev. Lett. 40, 1041 (1978); R. J. Watts-Tobin, Y. Krähenbühl, and L. Kramer, J. Low Temp. Phys. 42, 459 (1981).

${ }^{39}$ A. I. Gubin, K. S. Il'in, S. A. Vitusevich, M. Siegel, and N. Klein, Phys. Rev. B 72, 064503 (2005).

${ }^{40}$ R. Kato, Y. Enomoto, and S. Maekawa, Phys. Rev. B 47, 8016 (1993).

${ }^{41}$ M. Tinkham, Introduction to Superconductivity, 2nd ed. (McGrawHill, New York, 1996).

${ }^{42}$ G. R. Berdiyorov, M. V. Milošević, L. Covaci, and F. M. Peeters, Phys. Rev. Lett. 107, 177008 (2011).

${ }^{43}$ V. M. Krasnov, Phys. Rev. B 83, 174517 (2011).

${ }^{44}$ P. A. Warburton, S. Saleem, J. C. Fenton, M. Korsah, and C. R. M. Grovenor, Phys. Rev. Lett. 103, 217002 (2009).

${ }^{45}$ M. Beck, E. Goldobin, M. Neuhaus, M. Siegel, R. Kleiner, and D. Koelle, Phys. Rev. Lett. 95, 090603 (2005).

${ }^{46}$ J. B. Ketterson and S. N. Song, Superconductivity (Cambridge University Press, Cambridge, UK, 1999), p. 80.

${ }^{47}$ W. E. Lawrence and S. Doniach, in Proceedings of the 12th International Conference on Low Temperature Physics, Kyoto, 1970, edited by E. Kanda (Keigaku, Tokyo, 1970), p. 361. 